\title{
ESTUDIOS SOBRE LAS RUBIÁCEAS DE MÉXICO, XLIX Una nueva especie del género Arachnothryx (Rubiaceae, Guettardae) en la flora de Veracruz
}

\author{
A. Borhidi ${ }^{1}$, E. Martínez Salas ${ }^{2}$ y C. H. Ramos Álvarez ${ }^{3}$ \\ ${ }^{1}$ Departamento de Fitotaxonomía y Geobotánica, Instituto de Biología, Universidad de Pécs \\ H-7624 Pécs, Ifjúság útja 6, Hungría; E-mail: borhidi@gamma.ttk.pte.hu \\ ${ }^{2}$ Instituto de Biología, Universidad Autónoma de México (UNAM), México \\ E-mail:ems@ib.unam.mx \\ ${ }^{3}$ Av. IMAN 580, Helios 101, México, D. F., C.P. 4510, México \\ E-mail: clara.hilda.ramos@gmail.com
}

(Received 4 November, 2014; Accepted 15 December, 2014)

The new Arachnothryx species collected in Veracruz is a vicarious one of the next related Arachnothryx secundiflora (B. L. Rob.) Borhidi of Oaxaca, Tabasco and Chiapas, which differs in having sessile bractless cymes. Another closely related species A. linguiloba Borhidi et Diego Pérez of Guerrero differs in size and pubescence of stipules and leaves and the size and shape of the flowers. This paper presents also a short note about the generic delimitation of Arachnothryx Planch. 1849 not Lorence 2012.

Key words: Arachnothryx, Mexico, new, Rubiaceae

\section{Arachnothryx angustispica Borhidi, E. Martínez et Ramos, spec. nova, hoc loco}

(Figs 1-2)

Tipo: México, Estado Veracruz, Mpio. Tezonapa, Ejido San Antonio, conglomerado 64447 del Inventario Nacional Forestal y de Suelo. Selva alta perennifolia con vegetación secundaria arbustiva. Coord.: $18^{\circ} 70.1^{\prime} 11^{\prime \prime} \mathrm{N}, 96^{\circ} 87.5^{\prime} 56^{\prime \prime} \mathrm{W}$, alt. $1130 \mathrm{~m} \mathrm{snm}$. Col.: J. Canek Ledesma Corral y Vladimir Campos (JCLC 965), 22.10.2010. Holotipo: MEXU, isotipo: BP.

Observación: Las colectas fueron apoyados por los proyectos siguientes: Inventario Nacional Forestal de México: Colecta especial botánica 2010. Colecta de DIAAPROY S.A. de C.V. (México D.F.) por contrato con la Comisión Nacional Forestal (CONAFOR). Responsable: Martin Ricker Instituto de Biología UNAM, e-mail: mricker@ib.unam.mx.

Frutex ramosus usque ad 3 m altus. Rami cylindracei, cinerei, glabri, lenticellis dense rugosi, ramuli rubelli, adpresse brevi-pilosi vel glabri. Stipulae triangulares, acutae, 2-2.5 mm longae, extus glabrae, intus dense villosae, mucrone dorsali subter- 
minaliter disposito, 1-2 mm longo suffultae. Folia opposita, in uno nodo magnitudine inaequalia, sessilia vel subsessilia petiolo $0.5-3 \mathrm{~mm}$ longo anguste alato, adpresse sericeo vel glabro praedita, lanceolata, oblongo elliptica vel anguste oblonga, 3.2-10 $\mathrm{cm}$ longa et 1-3 cm lata, apice longe acuminata et saepe falcata, basi longe attenuata et decurrentia, concolora, viridia et utrinque opaca et sparse adpresse strigosa usque glabra; nervi laterales utroque latere 5-8, utrinque prominuli, ex vena centrali sub angulo acuto, arcuati et ante marginem anastomosantes. Inflorescentiae terminales, espiciformes, anguste oblongae, 6-13 cm longae et $1.8-2.5 \mathrm{~cm}$ latae, foliis breviores, sessiles vel pedunculis 5-15 mm longis, basi bracteis 2 foliaceis, $0.8-1.2 \mathrm{~cm}$ longis, bracteolae lineares, 4-5.5 $\mathrm{mm}$ longae, ante anthesim alabastra copriendae, strigososericeae. Flores bisexuales, magis distantes. Pedicelli 1-1.5 mm longi, dense albosericei. Hypanthium globosum, 1-1.2 mm longum et latum, albo-hirsutum. Calyx 4-merus, tubus $0.5 \mathrm{~mm}$ longus supra ovarium contractus, extus albo-hirsutus, intus glaber, lobi 4, inaequales, 3 minores, lineares, 2-3 mm longi, 1 maior, linear-obovatus usque linear-spathulatus, 3.5-5.5 mm longus, sub anthesi reflexus; omnes extus sericeo-hirsuti, intus glabri. Corolla rubra, 7-9 mm longa, tubus corollae 6-8 mm longus, basi 0.5-0.7 mm, fauce $2.5 \mathrm{~mm}$ latus, extus pilis antrorsis sericeo hirsutus, intus sub medio sericeo-puberulus; lobi 4, ovati usque obovati, 1.5-2 mm longi et 1-1.5 mm lati, in alabastra imbricati, exterior maior, margine undulato, extus sericei, intus glabri. Stamina 4, sessiles in $2 \mathrm{~mm}$ sub fauce glabro, antherae dorsifixae, lineares vel lineariellipticae, $2 \mathrm{~mm}$ longae et 0.3-0.4 mm latae. Stylus glaber, 7-8 mm longus, apice breviter 2-lobatus, leviter exsertus. Discus ovarii brevissimus, tomentosus. Fructus in pedicellis ascendentibus, 1-2 mm longis capsula globosa vel depresse globosa, 3-4 $\mathrm{mm}$ longa et 4-5 mm lata, adpresse sericea vel glabrescens, septicida. Placenta elliptica et convexa, $2 \mathrm{~mm}$ longa et $1 \mathrm{~mm}$ lata, foveolata ad septum supra medio inserta. Semina brunnea, angulosa et nitida, reticulato-foveolata, $0.5 \mathrm{~mm}$ longa et $0.3 \mathrm{~mm}$ lata.

Arbusto ramoso hasta $3 \mathrm{~m}$ de alto; ramas cilíndricas grisáceas glabras, muy densamente lenticelado-rugulosas, ramitas rojizas, apretado-corto-pelosas a glabras. Estípulas triangulares, agudas de 2 a $2.5 \mathrm{~mm}$ de largo, glabras por fuera, densamente vellosas por dentro, con un mucrón dorsal subterminal de 1 a $2 \mathrm{~mm}$ de largo. Hojas opuestas desiguales en tamaño en el par, sésiles o subsésiles con pecíolo de 0.5 a $3 \mathrm{~mm}$ de largo, estrechamente alado, apretado-seríceo a glabro; láminas lanceoladas, oblongo-elípticas o estrechamente oblongo elípticas, 3.2 a 10 por 1 a $3 \mathrm{~cm}$, largamente acuminadas y a menudo falcadas en el ápice, largamente atenuadas y decurrentes en la base, concoloras, verdes, mates, apretado estrigulosas o glabras en ambas caras, nervios laterales 5 a 8 pares, promínulos en ambas caras saliendo en ángulo agudo, arqueados y anastomosados antes del margen. Inflorescencias terminales más cortas que las hojas, sésiles o con pedúnculos de 5 a $15 \mathrm{~mm}$ de largo, con 2 brácteas foliáceas en la base, de 0.8 a $1.2 \mathrm{~cm}$ de largo, lineares y agudas, 


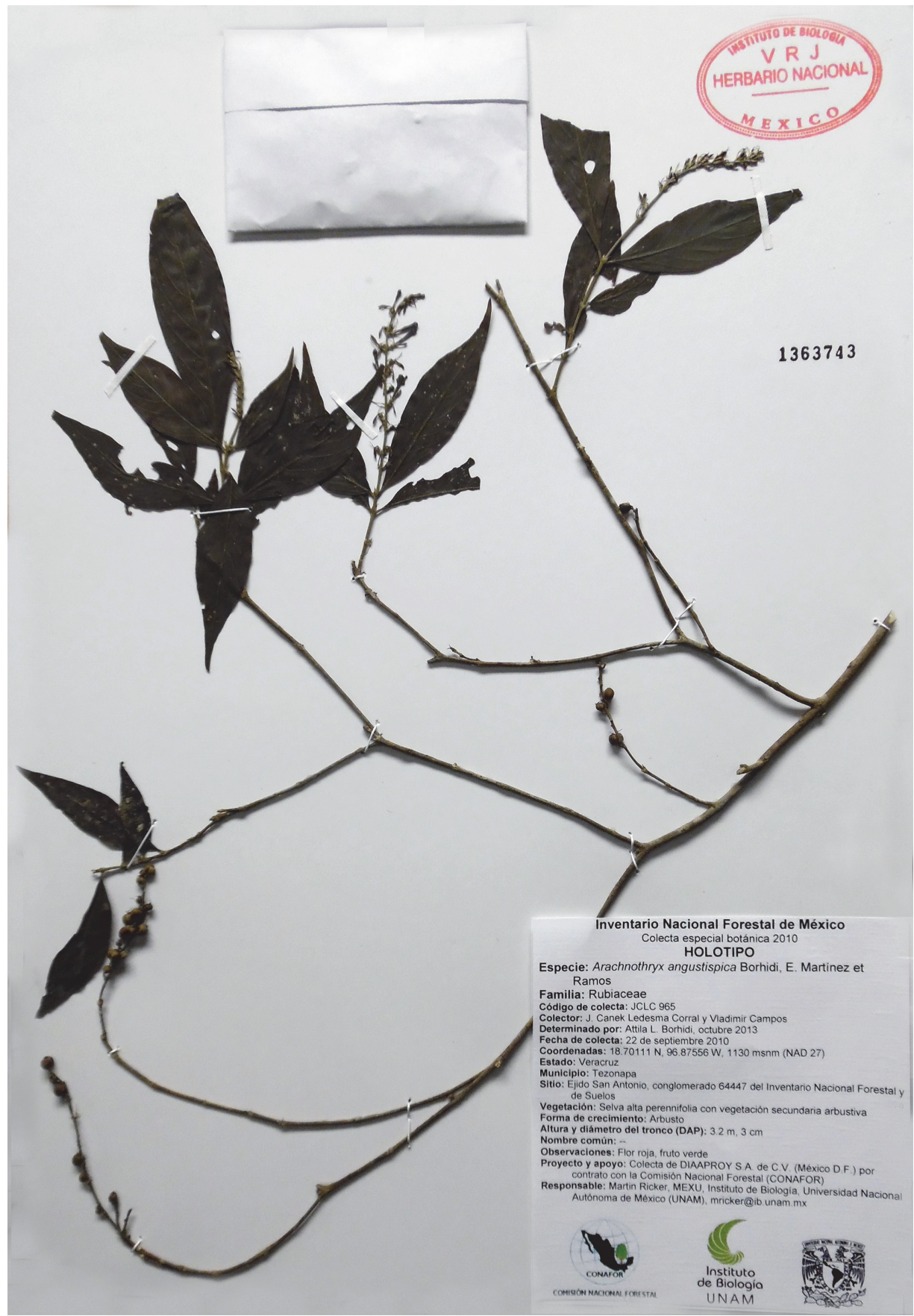

Fig. 1. Holotipo del Arachnothryx angustispica en MEXU (Foto: A. Borhidi) 


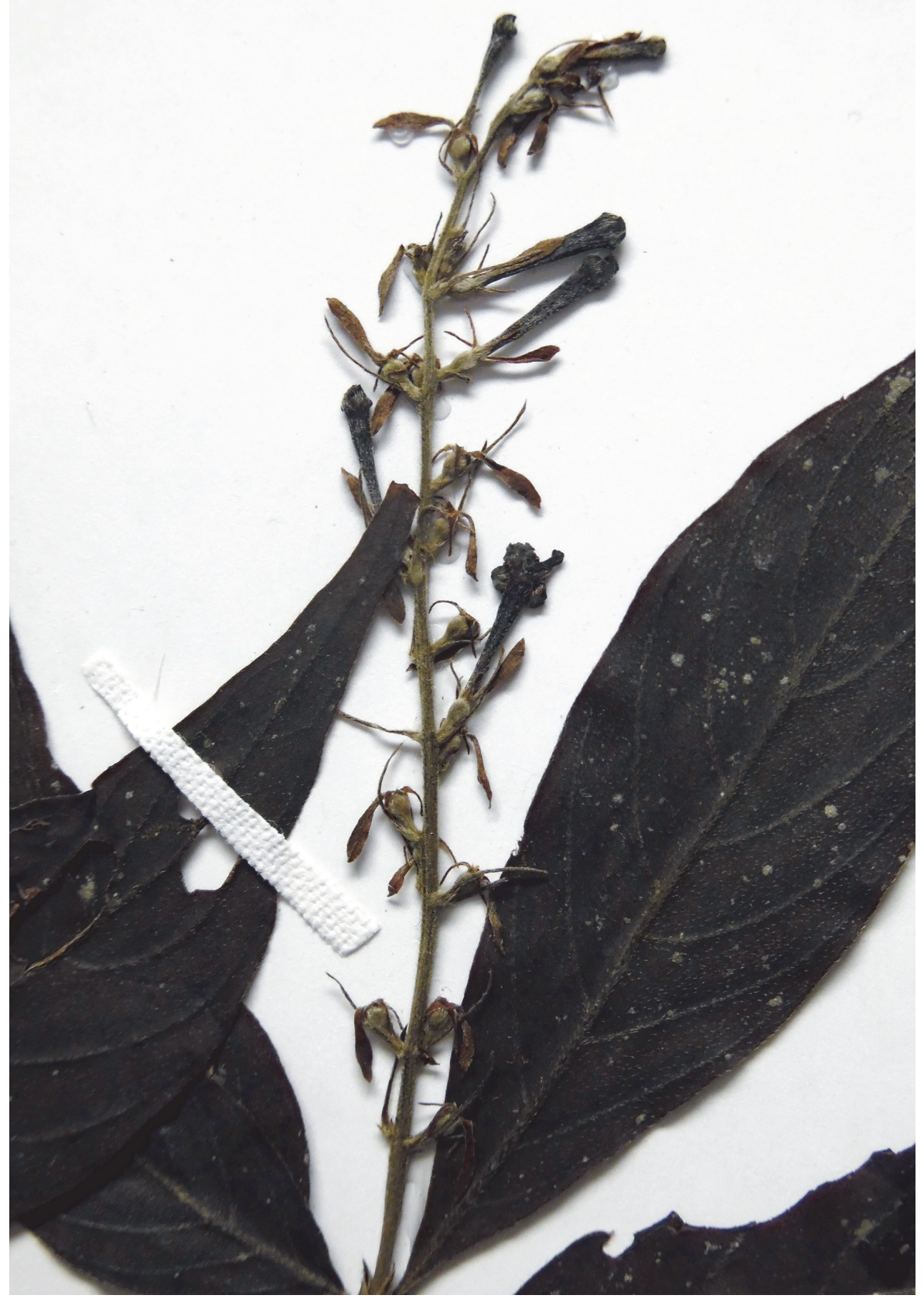

Fig. 2. Inflorescencia del Arachnothryx angustispica (Foto: A. Borhidi) 
formando una espiga, estrecha y alargada, bracteolata, de 6 a 13 por 1.8 a $2.5 \mathrm{~cm}$, más cortas que las hojas; bractéolas lineares de 4 a $5.5 \mathrm{~mm}$ de largo, antes de la floración ocultando los botónes florales, estriguloso-seríceas. Flores hermafroditas, probablemente distilas, sésiles o subsésiles, mayormente solitarias o en pares, las inferiores más espaciadas. Pedicelo de 1 a $1.5 \mathrm{~mm}$ de largo, densamente blanco-seríceo. Hipanto globoso de 1 a $1.2 \mathrm{~mm}$ de largo y ancho, blanco hirsuto. Cáliz 4-mero, tubo de $0.5 \mathrm{~mm}$ de largo estrechado sobre el ovario, blanco hirsuto por fuera, glabro por dentro, lóbulos 4 desiguales, 3 lineares, de 2 a $3 \mathrm{~mm}$ de largo, erguidos, el mayor linear-obovado a linear-espatulado, de 3.5 a $5.5 \mathrm{~mm}$ de largo, reflejo durante la floración; todos seríceo-hirsutos en el dorso, glabros en la cara ventral. Corola roja, de 7 a 9 $\mathrm{mm}$ de largo, tubo 6 a $8 \mathrm{~mm}$ de largo 0.5 a $0.7 \mathrm{~mm}$ de ancho en la base, $2.5 \mathrm{~mm}$ de ancho en la garganta, antrorso seríceo-hirsuto por fuera, seríceo-pubérulo en la mitad inferior por dentro; lóbulos 4 aovados a obovados de 1.5 a $2 \mathrm{~mm}$ de largo y 1 a $1.5 \mathrm{~mm}$ de ancho, imbricados en el botón, el exterior más grande y de margen ondulado, seríceos por fuera, glabros por dentro. Estambres 4, sésiles insertadas $2 \mathrm{~mm}$ debajo del borde de la garganta en la región glabra, anteras dorsifijas, linear-elípticas a lineares, $2 \mathrm{~mm}$ de largo y $0.3-0.4 \mathrm{~mm}$ de ancho. Estilo glabro, de 7 a $8 \mathrm{~mm}$ de largo, brevemente bilobado en el ápice, ligeramente exerto de la garganta. Disco del ovario muy corto. Fruto sobre pedicelos erguidos o ascendentes de 1 a $2 \mathrm{~mm}$ de largo una cápsula globosa $\mathrm{o}$ deprimido globosa, de 3 a $4 \mathrm{~mm}$ de largo y 4 a $5 \mathrm{~mm}$ de ancho, apretado seríceo a glabrescente, septicida. Placenta elíptica y convexa, de $2 \mathrm{~mm}$ de largo y $1 \mathrm{~mm}$ de ancho, foveolada, con una inserción al septo sobre la mitad. Semillas pardas, angulosas y nítidas, reticulado-foveoladas, de $0.5 \mathrm{~mm}$ de largo y 0.3 mm de ancho.

Afín al Arachnothryx linguiloba Borhidi et N. Diego-Pérez de Guerrero, que difiere de nuestra planta en tener estípulas bidentadas a bilobuladas con dos lóbulos lineares de hasta $4 \mathrm{~mm}$ de largo, estrigillosas por fuera y glabras por dentro; hojas del mismo por tamaño en el par, pecioladas con pecíolos de hasta $1 \mathrm{~cm}$ de largo, nervios laterales no conectados en el margen, domaciadas en el envés; inflorescencias más largas que las hojas superiores, con eje ferrugíneo-hirsuto, más anchas y compuestas por cimas laterales 3 a 5 -floras, lóbulos de 1 cáliz de 1 a $2 \mathrm{~mm}$ de largo, tubo de la corola más corto. Otra especie cercanamente relacionada es Arachnothryx secundiflora (B. L. Rob.) Borhidi con sus inflorescencias estrechas y largas, pero difiere de nuestra especie con tener hojas del mismo tamaño en el par, domaciadas en el envés, cimas laterales sésiles, bractéolas pequeñas o incospícuas, lóbulos del cáliz de 1-1.5 mm de largo, subiguales.

Nota: En la Flora Mesoamericana el género Arachnothryx esta tratado por D. H. Lorence (2012) en un sentido distinto de la descripción original de Plan- 
chon 1849, incluyendo varios elementos no pertenecientes a este género, ni a la tribu Guettardeae. Los estudios moleculares de Rova et al. (2009) y Manns y Bremer (2010) señalaron, que el género pertenece al tribu Guettardeae. Sin embargo Lorence incluye en Arachnotryx el Rondeletia deamii (Donn.-Sm.) Standl., en realidad Donnellyanthus deamii (Borhidi et al. 2011) perteneciente a Rondeletieae y las especies del género Renistipula (Borhidi et al. 2004) que pertenece a la tribu Hamelieae (Stranczinger et al. 2010, 2014).

Agradecimientos - Los autores expresan sus agradecimientos al Dr. Gerardo Adolfo Salazar, jefe del Departamento de Botánica, del Instituto de Biologia de la UNAM y al Dr. David Sebastian Gernandt, jefe del Herbario Nacional de México (MEXU) por su apoyo en crear condiciones favorables para este trabajo.

\section{LITERATURA CONSULTADA}

Borhidi, A. (2012): Rubiáceas de México. Segunda y ampliada edición. - Akadémiai Kiadó, Budapest, 610 pp.

Borhidi, A. y Diego-Pérez, N. (2009): Estudios sobre Rubiáceas Mexicanas XXI. Una nueva especies de Arachnothryx Planch. (Rondeletieae) en Guerrero. - Acta Bot. Hung. 51: 11-14. doi: 10.1556/ABot.51.2009.1-2.2

Borhidi, A., Darók, J. y Stranczinger, Sz. (2011): Donnellyanthus (Rubiaceae, Rondeletieae), a new genus in the flora of Mexico and Meso-America. - Acta Bot. Hung. 53: 273-281. doi: 10.1556/ABot.53.2011.3-4.9

Borhidi, A., Darók, J., Kocsis, M., Stranczinger, Sz. y Kaposvári, F. (2004): El Rondeletia complejo en México. - Acta Bot. Hung. 46: 91-135. doi: 10.1556/ABot.46.2004.1-2.8

Lorence, D. H. (2012): Arachnothryx Planch. - In: Davidse, G., Sousa, M., Knapp, S. y Chiang, F. (eds gen.): Flora Mesoamérica. IV(2): Rubiaceae, Cordiaceae, Boraginaceae, Lamiaceae. Universidad Nacional Autónoma de México, Missouri Botanical Garden, The Natural History Museum (London), pp. 16-37.

Manns, U. y Bremer, B. (2010): Towards a better understanding of intertribal relationships and stable tribal delimitations within Cinchonoideae s. s. (Rubiaceae). - Mol. Phyl. Evol. 56: 21-39. doi: 10.1016/j.ympev.2010.04.002

Rova, J. H. E., Delprete, P. G. y Bremer, B. (2009): The Rondeletia complex (Rubiaceae): An attempt to use ITS, rps16, and trnL-F sequence data to delimit Guettardeae, Rondeletieae and sections within Rondeletia. - Ann. Missouri Bot. Gard. 96: 182-193. doi: 10.3417/2006179

Stranczinger, Sz., Borhidi, A. y Szentpéteri, J. (2006): Phylogenetic relationships among some species of the Rondeletia complex (Rubiaceae). - Acta Bot. Hung. 48: 427-433. doi: 10.1556/ABot.48.2006.3-4.15

Stranczinger, Sz., Galambos, A. y Borhidi, A. (2010): Phylogenetic study on genus and species levels on the Deppea complex (Hamelieae section). - Abstracts, 5th Intern. Rubiaceae and Gentianales Conf., Stockholm, 6-10 September, p. 58.

Stranczinger, Sz., Galambos, A., Szenasy, D. y Szalontai, B. (2014): Phylogenetic relationships in the Neotropical tribe Hamelieae (Rubiaceae, Cinchonoideae) and comments on its generic limits. - J. Syst. Evol. 52(5): 643-650. doi: 10.1111/jse.12103 